\title{
Modelo para estimativa da produtividade para a cultura da soja
}

\author{
Model to estimate the yield for soybean
}

\section{Ana Carla dos Santos Gomes ${ }^{\mathrm{I}}$ Adroaldo Dias Robaina ${ }^{I I}$ Marcia Xavier Peiter ${ }^{\mathrm{II}}$ Fátima Cibele Soares ${ }^{\mathrm{III}}$ Ana Rita Costenaro Parizi ${ }^{\mathrm{I}}$}

\section{RESUMO}

Este trabalho teve por objetivo estimar e validar um modelo de simulação para a cultura da soja na região de Santiago, RS. Para isso, foi necessário desenvolver procedimento experimental, para geração de dados necessários a fim de calibrar e testar o modelo de simulação, da extração da água do solo, e a respectiva resposta em produção da cultura. Como resultados experimentais, obtiveram-se variações no crescimento e desenvolvimento da cultura da soja com a aplicação de diferentes estratégias de irrigação, resultando em produtividades distintas. $O$ aumento nas lâminas de irrigação proporcionou incremento nos componentes de produção. Os testes dos dados simulados demonstraram variação inferior a $8 \%$ na comparação dos dados medidos, no experimento de campo, com os dados simulados pelo modelo. O modelo de produção proposto foi capaz de simular satisfatoriamente o acúmulo de matéria seca total e a produção de grãos para a cultura da soja.

Palavras-chave: Glycine max (L.) Merrill, modelo de produção, estratégias de irrigação.

\section{ABSTRACT}

This study aimed estimate and validate a simulation model for the soybean crop in the region of Santiago, RS, Brazil which will, to the agricultural sector, support in decision making. For this, it was necessary to develop experimental procedure for generating the data needed to calibrate and test the simulation model, the extraction of water from the soil, and the response in crop production. As experimental results, obtained variations in the growth and development of soybean by applying different strategies for irrigation, resulting in different productivities. The increase in irrigation provided an increment in production components. Tests of simulated data showed a variation of less than $8 \%$ when comparing the data measured in field experiments with simulated data. The production model proposed was successfully able to simulate the accumulation of total dry matter and grain production for soybeans.

Key words: Glycine max (L.) Merrill, production model, irrigation strategies.

\section{INTRODUÇÃO}

O conhecimento prévio e preciso das safras agrícolas é uma questão estratégica para os produtores, bem como para o país, quer seja no planejamento do abastecimento interno (CORAL et al., 2005), quer para a orientação das ações referentes ao mercado externo, diminuindo a volatilidade do mercado (ZACHARIAS et al., 2008). Estimar safras agrícolas com precisão e antecipação é uma atividade complexa, em função da diversidade de cultivares encontradas no mercado, diferentes tipos de manejo, tipos de solos e/ou clima que são encontrados no Brasil (ALFONSI, 2008).

As previsões agrícolas constituem o meio mais importante de servir a agricultura. Grande parte das técnicas de previsão numérica baseia-se na agrometeorologia. Esta, por sua vez, fundamenta-se na relação estatística entre as variáveis dependentes que deverão ser estimadas, e as variáveis agrometeorológicas independentes (HOOGENBOOM et al., 2010).

Dessa maneira, a produtividade pode ser bem caracterizada por meio de modelos matemáticos

Instituto Federal Farroupilha (IFFarroupilha), Campus Alegrete, Alegrete, RS, Brasil.

IIDepartamento de Engenharia Rural, Centro de Ciências Rurais (CCR), Universidade Federal de Santa Maria (UFSM), Santa Maria, RS, Brasil.

IIIUniversidade Federal do Pampa (Unipampa), Campus Alegrete, 97546-550, Alegrete, RS, Brasil. E-mail:fatimasoares@unipampa.edu.br. Autor para correspondência. 
de monitoramento agrometeorológico. Esses modelos consideram que cada elemento climático exerce certo controle na produtividade da cultura, interferindo como um fator de eficiência (SILVA et al., 2011). Estes, além de fornecer dados para alimentar os sistemas de previsão de safras agrícolas, permitem identificar, ao longo do ciclo da cultura, fatores que atuam negativamente na produtividade, orientando a tomada de decisão (ROSA et al., 2010).

Os modelos de simulação são ferramentas que permitem analisar cenários, considerando as diversas combinações dos fatores que influenciam a produtividade das culturas. Dessa forma, é possível avaliar as estratégias mais adequadas em cada condição específica, podendo modificar a estratégia de irrigação para predizer as alterações nos componentes de produção e de outras variáveis, como a evapotranspiração e as necessidades de água da cultura (PARIZI, 2010).

Para o processo de planejamento, esses modelos constituem o elemento básico de decisão dos planos de desenvolvimento e, quanto à operação de projetos de irrigação, permitem tomar decisões sobre planos ótimos de cultivo e ocupação de área para produção econômica com base na água disponível. Possibilitam, também, a escolha correta da época de plantio, para que a cultura não fique exposta a déficits hídricos em momentos cruciais à obtenção de uma boa produção (HOWELL et al., 1992; FRIZZONE et al., 2005).

CABELGUENNE \& JONES (1989) e LAL et al. (1993) utilizaram modelos de simulação para definir as estratégias de irrigação e de manejo, bem como estudo da viabilidade econômica das culturas. A programação da irrigação para a cultura da soja exige o conhecimento de métodos para determinar o tempo de aplicação de água. Em uma análise agroclimatológica da necessidade de irrigação da soja no Rio Grande do Sul, MOTA et al. (1996) concluíram que, em todas as regiões do Estado, há necessidade de irrigação, considerando o período de semeadura recomendado e as cultivares.

Nesse sentido, o conhecimento de funções de produção da cultura da soja permite auxiliar no manejo de estratégias de irrigação, trazendo informações importantes para os produtores da região centro-oeste do Estado do Rio Grande do Sul. Desse modo, o trabalho teve por objetivo estimar e validar um modelo de simulação para a cultura da soja na região de Santiago, RS.

\section{MATERIAL E MÉTODOS}

O experimento de campo, com a cultivar de soja CD 219 RR, foi conduzido na safra agrícola 2008/2009, no município de Santiago-RS, em Latossolo Vermelho Distrófico típico (EMBRAPA, 1999), em área experimental situada na latitude $29^{\circ}$ 09' "50" Sul, longitude 54 51 " 32 " Oeste, a 439m de altitude, com temperatura média de $17,9^{\circ} \mathrm{C}$ e precipitação anual de 1.919mm (GOMES, 2007).

O modelo físico utilizado para calibração e validação do modelo matemático foi constituído de seis estratégias de irrigação (tratamentos), distribuídas em um sistema de irrigação por aspersão convencional, constituído por uma linha principal e seis linhas laterais fixas, todas de PVC e diâmetro de $50 \mathrm{~mm}$. O momento de aplicação da irrigação foi baseado no turno de rega prefixado, com intervalo de sete dias entre as irrigações, quando não ocorria precipitação pluviométrica.

A quantidade de água a irrigar foi determinada através da medida diária da evaporação da água (Tanque Classe A) e o volume de água a ser aplicada na irrigação $\left(\mathrm{V}_{\mathrm{i}}\right)$, sempre que o sistema era posto em funcionamento, foi calculado através da expressão: $V_{i}=K p \cdot \sum_{i=1}^{n=7} E_{T A}$, em que, $\mathrm{V}_{\mathrm{i}}$ é o volume de água para a irrigação (mm), $\mathrm{E}_{\mathrm{TA}}$ é a evaporação do Tanque Classe A e Kp é o coeficiente do tanque, cujo valor médio é igual a 0,7 .

O tempo de funcionamento $(\mathrm{h})$ do sistema de irrigação para aplicar a lâmina de água necessária foi determinado a partir da intensidade de aplicação de água $\left(\mathrm{mm} \mathrm{h}^{-1}\right)$ do sistema de irrigação.

Para a determinação da intensidade de aplicação de água, o sistema foi posto para funcionar durante uma hora, sendo a chuva artificial recolhida por pluviômetros dispostos em toda a área. Os volumes de água coletados nos pluviômetros, no intervalo de uma hora, foram convertidos em lâminas d' água, no setor de irrigação (T5), que correspondia a $100 \%$ da água aplicada. A partir dessa informação, foram colocados registros na entrada de cada linha principal. Os registros foram regulados a fim de distribuírem lâminas de menor intensidade.

Os tratamentos foram definidos como: 20\% (Tratamento 1 - T1) da evapotranpiração de referência (Eto), 40\% (Tratamento 2 - T2) da Eto, $60 \%$ (Tratamento 3 - T3) da Eto, 80\%, (Tratamento 4 - T4) da Eto, e 100\% (Tratamento 5 - T5) da Eto estimada por um tanque classe A. Analisou-se também a testemunha (Tratamento 0 - T0) com $0 \%$ da Eto, que recebeu somente a precipitação ocorrida no período. 
O modelo matemático utilizado foi o ROBAINA (2009 - Informe verbal). Ele leva em consideração as características do solo, da atmosfera e da planta para acompanhar a extração de água no solo, e a resposta da produção da cultura sob diferentes manejos de irrigação.

Foi utilizada a equação de RICHARDS (1931) para descrever as variações do potencial matricial da água no solo, modificada com a inclusão de um termo representativo da transpiração, expressa por:

$\frac{\partial \psi}{\partial t} C(\psi)=\frac{\partial}{\partial z}\left[k(\psi) \cdot \frac{\partial \psi}{\partial z}+k(\psi)-\mathrm{T} R(\psi)\right]$, em que: $C(\psi)$ é a variação do potencial matricial em função da variação de umidade volumétrica do solo, $T R(\psi)$, é a transpiração da planta em função do potencial matricial, cujo valor varia na profundidade $z$ do solo e no tempo t, $k(\psi)$ é a condutividade hidráulica do solo não saturado.

A estimativa da transpiração real foi calculada por: $\operatorname{TR}(\psi)=T P_{\text {máx }} \cdot f_{T P} \cdot f_{S R}$, em que: $\mathrm{TP}_{\text {máx }}$ é a transpiração máxima $\left(\mathrm{mm}\right.$ dia $\left.^{-1}\right)$. A redução da taxa de transpiração, devido à redução do potencial matricial $(\psi)$ na profundidade $z$ e no tempo $t$, foi definida por: $f_{T P}=\frac{\psi(z, t)-\psi_{p m}(z)}{\Psi_{c c}(z)-\Psi_{p m}(z)}$, em que os potenciais matriciais $\psi_{c c}(z)$ e $\psi_{p m}(z)$ são os que correspondem à umidade do solo em capacidade de campo e a umidade do solo no ponto de murcha permanente na camada $z$; e $f_{S R}$ é uma função que representa a distribuição do sistema radicular no tempo e em diferentes profundidades no perfil do solo. A distribuição do sistema radicular, que representa a extração da água nas diferentes profundidades no perfil do solo, foi calculada por: $f_{S R}=\frac{g(z)}{\int_{0}^{\operatorname{Pr} z} g(z)}$, em que: $f_{S R}$ é a fração do sistema radicular de profundidade $\operatorname{Pr} z$ até a profundidade $\mathrm{z}$. O valor da função $\mathrm{g}(\mathrm{z})$ foi calculado por: $g(z)=\frac{c \cdot(2 \cdot z-\operatorname{Pr} z)+\operatorname{Pr} z}{\operatorname{Pr} z^{2}}$, sendo c uma constante, em que se adotou o valor de $-0,8$ (PERROCHET, 1987); Pr $z$ é a profundidade do sistema radicular, sendo calculada por:

$\operatorname{Pr} z=a+\frac{b}{1+e^{\frac{D A E-c}{d}}}$, em que as constantes

( $\mathrm{a}, \mathrm{b}, \mathrm{c}, \mathrm{d})$ foram determinadas por ajustamento aos dados da profundidade das raízes, medidos nas trincheiras abertas para essa finalidade no experimento no campo.

A previsão de produção de grão da cultura de soja foi feita em função da produção de matéria seca da parte aérea da cultura e do índice de colheita. Segundo ROBAINA (1992), o modelo de produção de matéria seca da parte aérea da cultura pode ser separado em submodelo para a estimativa da produção potencial da cultura e submodelo para a estimativa da produção real da cultura.

A produção potencial diária de matéria seca $\left(q_{\mathrm{p}}\right)$, para um dia qualquer, em condições de campo, foi expressa por:

$$
q_{P}=\left[\eta \cdot P_{o}+(1-\eta) \cdot P_{C}\right] \cdot \alpha \cdot \beta \cdot \lambda \cdot \frac{I A F}{5} \text {, em que: }
$$

$q_{\mathrm{p}}\left(\mathrm{kg} \mathrm{ha}^{-1}\right.$ dia $\left.^{-1}\right), \eta$ é a fração do dia em que o céu está nublado, $P_{o}$ é a taxa de matéria seca para dias nublados, $P c$ é a taxa de matéria seca para dias claros, ambos expressos em $\mathrm{kg} \mathrm{ha}^{-1}$ dia $^{-1}$ e funções da latitude do local e da época do ano, $\alpha$ é o fator de influência da temperatura, $\beta$ é a relação entre a massa seca total da planta sem raízes e a massa seca total da planta com raízes, $\lambda$ o fator de respiração e IAF é o índice de área foliar $\left(\mathrm{m}^{2} \mathrm{~m}^{-2}\right)$.

O valor da fração do dia em que o céu está nublado, segundo De WIT (1978), foi determinado

por $\eta=1,25-0,625 \cdot \frac{R s}{R c}$, na qual Rc é a radiação

fotossinteticamente ativa na ausência da atmosfera $\left(\mathrm{MJ} \mathrm{m}^{-2} \mathrm{dia}^{-1}\right)$, Rs é a radiação solar global média ao nível do solo $\left(\mathrm{MJ} \mathrm{m}^{-2}\right.$ dia $\left.^{-1}\right)$, determinada através da estação de coleta de dados agrometeorológicos.

O fator de influência da temperatura $(\alpha)$ sobre a produção potencial diária foi obtido por meio da seguinte equação: $\alpha=1-\frac{T_{m}-T_{L I}}{T_{L S}-T_{L I}}$, em que: $\mathrm{T}_{\mathrm{LI}}$ refere-se a temperatura limite inferior, sendo considerada igual a $20^{\circ} \mathrm{C}$ e $\mathrm{T}_{\mathrm{LS}}$ se refere à temperatura limite superior, sendo igual a $35{ }^{\circ} \mathrm{C}$ para a soja. $\mathrm{O}$ fator de redução da produção devido à respiração $(\lambda)$ foi considerado, neste estudo, igual a 0,30 , conforme FEDDES (1976).

A relação entre a massa seca total da planta sem as raízes e a massa seca total da planta com as raízes, simbolizado por $\beta$, foi considerado, neste estudo, igual a 0,92, segundo FEDDES (1976).

$\mathrm{O}$ índice de área foliar, nos diferentes

tratamentos, foi calculado por: $I A F=a \cdot e^{-0.5\left(\frac{D A E-b}{c}\right)^{2}}$, em que as constantes $(\mathrm{a}, \mathrm{b}, \mathrm{c})$ foram determinadas por ajustamento aos dados do índice de área foliar medidos no campo.

A produção potencial acumulada $\left(Q_{p}\right)$, ao longo do ciclo de crescimento da cultura, foi estimada por: $Q_{P}=\sum_{1}^{n} q_{P} . \Delta t$, em que: $Q_{p}$ é obtido em $\mathrm{kg} \mathrm{ha}^{-1}, n$ é 
o período de dias até a maturação da cultura (colheita) e $\Delta t$ o período de 1 dia.

A estimativa da produção real diária de matéria seca da parte aérea de uma cultura, em função do número de dias, segundo ROBAINA (1992), foi estimado por meio de:

$q_{R}^{i}=\frac{q_{p}^{i}}{2}+A \frac{T R^{i}}{2 \Delta e^{i}}-\frac{1}{2} \sqrt{\left(q_{p}^{i}+A \cdot T R^{i} / \Delta e^{i}\right)^{2}}-4 \cdot(1-\varepsilon) \cdot q_{p}^{i} \cdot A \cdot \frac{T R^{i}}{\Delta e^{i}}$, em que o termo $q_{\mathrm{R}}$ é expresso em $\mathrm{kg} \mathrm{ha}^{-1} \mathrm{dia}^{-1}, \Delta$ e é o déficit de pressão de vapor da água $(\mathrm{hPa})$. Os valores de $\mathrm{A}$ e $\varepsilon$ foram determinados na fase de calibração do modelo de simulação da extração da água do solo pelas raízes (transpiração) e da produção da cultura de soja.

A produção real acumulada $\left(Q_{R}\right)$ foi calculada pela soma das produções diárias durante todo o período, através da seguinte expressão:

$Q_{R}=\sum_{1}^{n} q_{R} \cdot \Delta t$ , em que: $Q_{R}$ é obtido em $\mathrm{kg} \mathrm{ha}^{-1}, n$ é o período de dias até a maturação da cultura (colheita) e $\Delta t$ o período de 1 dia.

A produção de grãos com $13 \%$ de umidade da cultura $(P g c)$, obtida pelo modelo computacional, foi calculada por: $P g c=1,15 \cdot Q_{R} . I C$, em que: $Q_{R}$ é a produção de matéria seca $\left(\mathrm{kg} \mathrm{ha}^{-1}\right)$ e $I C$ é o índice de colheita, determinado a partir dos dados dos valores obtidos no experimento de campo (modelo físico experimental) e 1,15 é o fator de correção da umidade do grão.

A calibração do modelo de produção se refere à determinação dos coeficientes $A$ e $\varepsilon$. Foram utilizados os valores de produção obtidos no tratamento T0 para realizar esse procedimento. O teste do modelo de simulação foi feito com os valores de produção obtidos no experimento de campo nos tratamentos T1, T2, T3, T4 e T5, e os valores de produção calculados com o modelo matemático calibrado.

Para interpretação dos resultados, foi determinado o coeficiente de correlação (r) (SCHNEIDER, 1998) e índice de concordância (c) de Willmott. A avaliação do desempenho do modelo de produção da soja foi feita pelo índice de desempenho (id), proposto por CAMARGO \& SENTELHAS (1997), cujo valor é o produto do coeficiente de correlação e o índice de concordância.

\section{RESULTADOS E DISCUSSÃO}

Observou-se que os valores das variáveis, que participam do modelo de produção proposto: radiação solar fotossinteticamente ativa $(\mathrm{Rc})$, taxa de produção potencial nos dias claros $(\mathrm{Pc})$ e taxa de produção potencial nos dias nublados (Po), obtidos a partir dos dados de DOORENBOS \& KASSAM (1994) diminuem à medida que se aproximam da metade do ano. Eles voltam a crescer a partir da metade do ano, de modo a se aproximarem do valor do início do ano, sugerindo que uma função do tipo senoidal se ajustaria adequadamente aos dados, de modo a permitir o cálculo dos valores de qualquer variável e em qualquer dia do ano.

Para a calibração do modelo, foram utilizados os valores de produção de matéria seca total e os valores de produção de grãos, obtidos no tratamento T0. O processo de calibração foi concluído quando a diferença entre os valores de produção de matéria seca, obtidos por intermédio do modelo, e os valores de produção de matéria seca obtidos no experimento de campo foi o menor possível. O resultado final do processo de calibração apresentou o valor $A=42,5$ e $\varepsilon=0,01$. Observa-se que a diferença na produção de matéria seca e de grãos, para a cultivar estudada, foi de $3,4 \%$ e $1,3 \%$, respectivamente (Tabela 1).

O teste do modelo refere-se à avaliação da capacidade de fornecer a previsão da produção de matéria seca e de grãos. Após a calibração do modelo, pelo Tratamento T0, realizou-se o teste da capacidade deste em reproduzir os valores de produção para os demais tratamentos. Para verificar o desempenho do modelo de produção, os valores da produção de matéria seca e da produção de grãos, obtidos com a utilização do modelo de produção, foram comparados com os valores da produção de matéria seca e da produção de grãos, obtidos em experimento de campo. Observou-se que a diferença entre os valores da produção de matéria seca variaram de 4,6 a $7,1 \%$, sendo que os valores de produção de grãos apresentaram variação de $-0,2$ a 7,2\% (Tabela 1). $\mathrm{O}$ Tratamento T3, para a produção de matéria seca, foi o que apresentou menor variação $(4,6 \%)$. No que se refere à produção de grãos, o tratamento $\mathrm{T} 4$ foi o que apresentou menor variação $(-0,2 \%)$.

Esses valores corroboram os encontrados por FERREIRA (2007), que testou um modelo agrometeorológico para estudo da influência da variabilidade climática na cultura da soja. MORAES et al. (1998), ao avaliar o desempenho de modelos agrometeorológicos, baseados na penalização da produtividade por deficiência hídrica para algumas safras de soja na região de Ribeirão Preto, SP, constataram que o modelo proposto por DOORENBOS \& KASSAM (1979), o mesmo incorporado ao SPSoja-Agritempo, também tendeu a superestimar a produtividade medida em campo. 
Tabela 1 - Calibração dos valores de produção de matéria seca e de grãos obtidos após a calibração do tratamento (T0) para as duas cultivares de soja (A). Comparação dos valores de produção de matéria seca total e de produção de grãos obtidos pelo modelo de produção e no experimento de campo para a cultivar de soja (B). Valores do coeficiente linear (a), coeficiente angular (b), coeficiente de determinação $\left(\mathrm{R}^{2}\right)$, coeficiente de correlação (r), índice de concordância (c) e índice de desempenho (id), obtidos na comparação entre a produção de matéria seca e de grãos, obtidos em experimento de campo e pelo modelo de produção, para os diferentes tratamentos $(\mathrm{C})$.

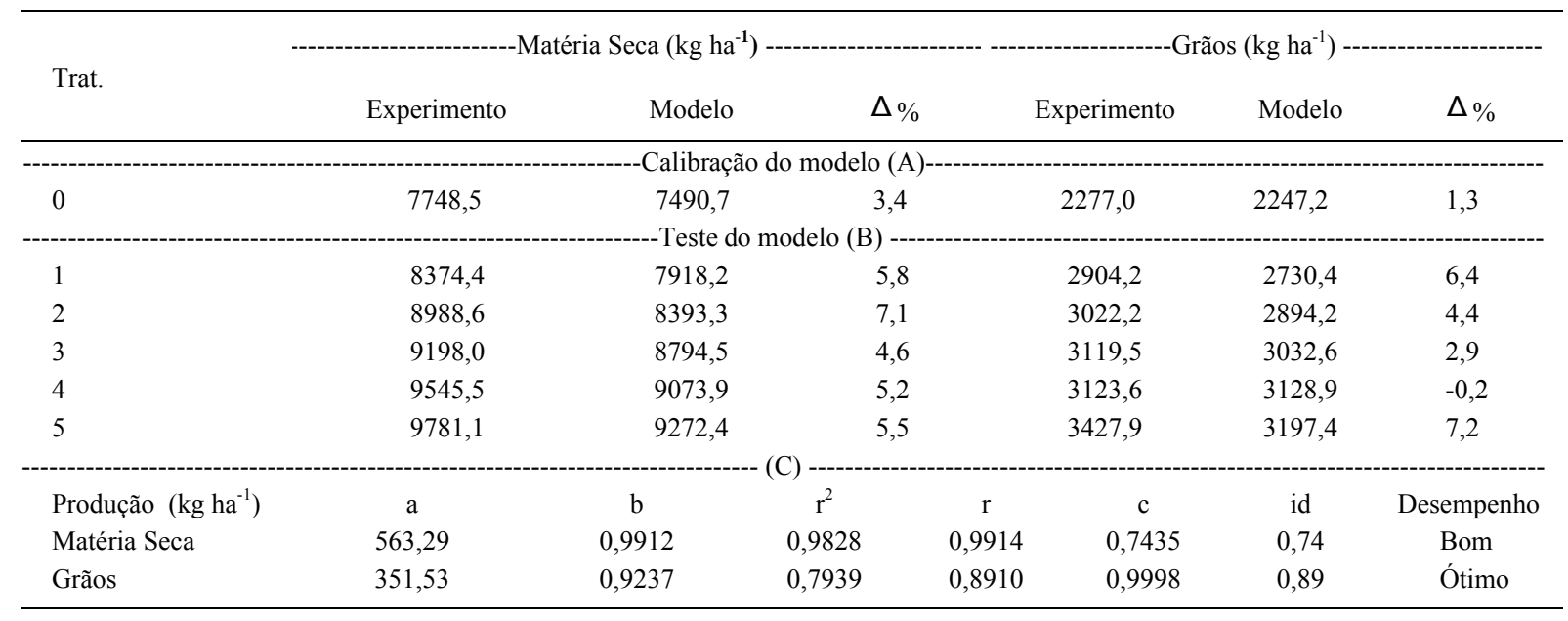

ASSAD et al. (2007) utilizaram este modelo na safra 2003/2004 e concluíram que houve subestimativa de $12,5 \%$, na Região Sul, ano em que o SPSojaAgritempo apresentou sensibilidade excessiva à deficiência hídrica, o que penaliza em demasia a produtividade.

Para reforçar a validação do modelo de produção, foram determinados os coeficientes lineares e angulares da reta de regressão, dos valores de matéria seca e de grãos, obtidos por simulação (x), e os valores de matéria seca, obtidos no experimento de campo (y). Os resultados podem ser observados na tabela 1 , na qual consta também o coeficiente de determinação $\mathrm{R}^{2}$.

Verificou-se que os valores do coeficiente de correlação foram maiores que 80\% (Tabela 1), evidenciando a forte correlação entre as duas formas de obtenção (modelo ou campo) dos parâmetros que foram comparados (matéria seca total e de grão).

Os valores do índice de concordância foram de aproximadamente 74 e 100\%, para matéria seca e produção de grãos, respectivamente. Esse índice mostra uma concordância muito forte entre os valores da produção de grãos. Os valores do índice de desempenho variaram entre 74 e $89 \%$, evidenciando que os valores de matéria seca e de grão, obtidos pelo modelo de simulação, apresentaram uma forte aderência, aos valores de matéria seca e de grãos, obtidos no experimento de campo. As classes de desempenho obtidas foram "bom" e "ótimo", para matéria seca e produção de grãos, respectivamente.
Os resultados obtidos demonstram que o modelo proposto é perfeitamente viável, e que pode ser utilizado como uma ferramenta para estimar a produtividade de grãos de uma cultura da soja. Esses resultados são semelhantes aos de DOURADO NETO et al. (2005), quando propuseram modelos matemáticos-fisiológicos para estimar o acúmulo de matéria seca dos diferentes órgãos da parte aérea e produtividade de grãos de milho. Os autores observaram que os modelos propostos foram precisos na estimativa do acúmulo de matéria seca, nos diversos órgãos da parte aérea das plantas de milho, bem como para prever o rendimento de grãos da cultura. RODRIGUES (2012), avaliando a necessidade de adubação nitrogenada, para a produção de grãos de milho, através de modelos determinísticos, concluiu que a planta apresentou "ótima" capacidade preditiva, podendo ser utilizado para simulação de produtividade de grãos da cultura, assim como para estimar as exigências em nitrogênio para produção.

\section{CONCLUSÃO}

O modelo proposto é capaz de prever satisfatoriamente o rendimento de grãos da cultura, tornando-o, assim, uma importante ferramenta na previsão da produtividade em condições irrigadas, evidenciando as melhores estratégias de irrigação que resultem em elevadas produções de grãos. 


\section{INFORME VERBAL}

ROBAINA, A.D. diasrobaina@gmail.com Departamento de Engenharia Rural, Centro de Ciências Rurais (CCR), Universidade Federal de Santa Maria (UFSM). Av. Roraima, $\mathrm{n}^{\circ}$ 1000. Santa Maria - RS, 97105-900, Brasil. Programa computacional MFLUXO. Comunicação pessoal, em 28 jun. 2009.

\section{REFERÊNCIAS}

ALFONSI, E.L. Uso de índices fenológicos em modelos de previsão de produtividade do cafeeiro. 2008. 104f. Tese (Doutorado em Agronomia) - Curso de Pós-graduação em Agronomia, Escola Superior de Agricultura Luiz de Queiroz, Piracicaba, SP.

ASSAD, E.D. et al. Sistema de previsão de safra de soja para o Brasil. Pesquisa Agropecuária Brasileira, v.42, n.5, p.615-625, 2007. Disponível em: <http://www.scielo.br/pdf/pab/v42n5/02. pdf>. Acesso em: 12 dez. 2012.

CABELGUENNE, M.; JONES, C.A. Simulation of cropping systems in Southern France. In: SUMMER COMPUTER SIMULATION CONFERERENCE, 1989, Austin, Texas. Proceedings... Austin, Texas: The Society, 1989. p.707-711.

CAMARGO, A.P.; SENTELHAS, P.C. Avaliação do desempenho de diferentes métodos de estimativa da evapotranspiração potencial no estado de São Paulo, Brasil. Revista Brasileira de Agrometeorologia, v.5, n.1, p.89-97, 1997.

CORAL, A. et al. Utilização de um modelo agrometeorológico na estimativa de produtividade da cultura da soja no Estado do Paraná. In: CONGRESSO BRASILEIRO DE AGROMETEOROLOGIA, 14., 2005, Campinas, SP. Anais... Campinas: Sociedade Brasileira de Agrometeorologia, 2005. (CD ROM).

DOORENBOS, J.; KASSAM, A.H. Yield response to water. Roma: FAO, 1979. 193p

.; KASSAM, A.M. Efeito da água no rendimento das culturas. Campina Grande: UFPB, 1994. 306 p.

DOURADO NETO, D. et al. Modelos matemáticos-fisiológicos para estimar matéria seca da parte aérea e produtividade de grãos de milho. Revista brasileira Agrociência, v.11, n.4, p.409-418, 2005. Disponível em: <http://www.ufpel.edu.br/faem/agrociencia/ v11n4/artigo04>. Acesso em: 12 dez. 2012.

EMPRESA BRASILEIRA DE PESQUISA AGROPECUÁRIA (EMBRAPA). Centro Nacional de Pesquisa de Solos. Sistema brasileiro de classificação de solos. 2.ed. Rio de janeiro: Embrapa-CNPS, 1999. 412p.

FEDDES, R.A. Simulation of field water uptake by plants using a soil water dependent root extraction function. Journal of Hydrology, v.1, p.13-26, 1976. Disponível em: <http://www. sciencedirect.com/science/article/pii/0022169476900172>. Acesso em: 12 dez. 2012. doi: 10.1016/0022-1694(76)90017-2.

FERREIRA, W.P.M. Teste de um modelo agrometeorológico para estudo da influência da variabilidade climática na cultura da soja. Revista Ceres, v.54, n.312, p.206-213, 2007.
Disponível em: <http://www.ceres.ufv.br/CERES/revistas/ V54N312P03007.pdf>. Acesso em: 12 dez. 2012.

FRIZZONE, J.A. et al. Funções de produção água-cultura. In: et al. (Ed.). Planejamento de irrigação: análise e decisão de investimento. Brasília: Embrapa, 2005. p.283-314.

GOMES, A.C.S. Efeito de diferentes estratégias de irrigação sob a cultura da soja (Glycine max (L.) Merril) na região de Santiago, RS. 2007. 132f. Dissertação (Mestrado em Engenharia Agrícola) - Curso de Pós-graduação em Engenharia Agrícola, Universidade Federal de Santa Maria, RS.

HOOGENBOOM, G. et al. Decision support system for agrotechnology transfer (DSSAT) Version 4.5. Honolulu: University of Hawaii, 2010. 1 CD-ROM.

HOWELL, T.A. et al. Crop yield response. In: HOFFMAN, G.J. et al. (Ed.). Management of farm irrigation of agricultural systems. St. Joseph: American Society of Agricultural Engineers, 1992. p. $93-122$

LAL, H. et al. Using crop simulation models and GIS for regional productivity analysis. Trans. ASAE, v.36, p.175-184, 1993. Disponível em: <http://elibrary.asabe.org/toc_journals.asp?volum $\mathrm{e}=36$ \& issue $=1$ \& conf $=\mathrm{t}$ \&orgconf $=\mathrm{t} 1993>$. Acesso em: 7 jan. 2012 .

MORAES, A.V.C. et al. Teste e análise de modelos agrometeorológicos de estimativa de produtividade para a cultura da soja na região de Ribeirão Preto. Bragantia, v.57, n.2, p.393406, 1998. Disponível em: <http://www.iac.sp.gov.br/publicacoes/ bragantia/volumesonline.php>. Acesso em: 7 jan. 2012. doi: 10.1590/S0006-87051998000200021.

MOTA, F.S. et al. Análise agroclimatológica da necessidade de irrigação da soja no Rio Grande do Sul. Revista Brasileira de Agrometeorologia, v.4, n.1, p.133-138, 1996. Disponível em: $<$ http://coral.ufsm.br/rba/t13313841.html >. Acesso em: 7 jan. 2012.

PARIZI, A.R.C. Funções de produção das culturas de milho e feijão através de estudo experimental e simulado. 2010. 205f. Tese (Doutorado em Engenharia Agrícola) - Curso de Pósgraduação em Engenharia Agrícola, Universidade Federal de Santa Maria, RS.

PERROCHET, P. Water uptake by plant roots $-\mathrm{A}$ simulation model: conceptual model. Journal of hydrology, v. 95, p.55-61. 1987.

RICHARDS, L.A. Capillary conduction of liquids through porous médiuns. Physics, v.1, p.318-333, 1931.

ROBAINA, A.D. Estudo experimental e de simulação numérica da aplicação da água na produção das culturas. 1992. 144f. Tese (Doutorado em Recursos hídricos e Saneamento) - Curso de Pós-graduação em Engenharia Civil, Escola de Engenharia de São Carlos, SP.

RODRIGUES, M. da S. Modelo determinístico para estimativa da adubação nitrogenada na cultura do milho para grãos e silagem. 2012. 98f Dissertação (Mestrado em Agronomia)Curso de Pós-graduação em Agronomia, Universidade Tecnológica Federal do Paraná, PR.

ROSA, V.G.C. et al. Estimativa da produtividade de café com base em um modelo agrometeorológicoespectral. Pesquisa Agropecuária Brasileira, v.45, n.12, p.1478-1488, 2010. 
Disponível em: <http://seer.sct.embrapa.br/index.php/pab/article/ view/8623>. Acesso em: 7 jan. 2012.

SCHNEIDER, P.R. Análise de regressão aplicada à engenharia florestal. Santa Maria: UFSM/CEPEF, 1998. 236p.

SILVA, S, de A. et al. Modelo agrometeorológico na estimativa da produtividade de duas variedades de café arábica considerando a variabilidade espacial. Irriga, v.16, n.1, p.1-10, 2011. Disponível em: <http://irriga.fca.unesp.br/index.php/irriga/article/view/186>. Acesso em: 7 jan. 2012.

ZACHARIAS, A.O. et al. Modelo agrometeorológico de estimativa do início da florada plena do cafeeiro. Bragantia, v.67, n.1, p.249-256, 2008. Disponível em: <http://www.scielo.br/scielo. php?script $=$ sci_arttext\&pid=S0006-87052008000100030\&lng=pt \&nrm=iso >. Acesso em: 14 jan. 2012. doi.org/10.1590/S000687052008000100030 